Supplement of Biogeosciences, 15, 6185-6198, 2018

https://doi.org/10.5194/bg-15-6185-2018-supplement

(C) Author(s) 2018. This work is distributed under

the Creative Commons Attribution 4.0 License.

(c) (1)

Supplement of

\title{
The distinct roles of two intertidal foraminiferal species in phytodetrital carbon and nitrogen fluxes - results from laboratory feeding experiments
}

Julia Wukovits et al.

Correspondence to: Julia Wukovits (julia.wukovits@univie.ac.at)

The copyright of individual parts of the supplement might differ from the CC BY 4.0 License. 
Table S1. Raw data of EA-IRMA of foraminiferal samples of Paper 4 (n.a. = natural abundance, data for 5.5 P from Paper 3).

\begin{tabular}{lrrrr} 
& $\mathrm{d} 15 \mathrm{~N} / 14 \mathrm{~N}$ & $\mathrm{AT} \% 15 \mathrm{~N} / 14 \mathrm{~N}$ & $\mathrm{~d} 13 \mathrm{C} / 12 \mathrm{C}$ & \multicolumn{2}{c}{$\mathrm{AT} \% 13 \mathrm{C} / 12 \mathrm{C}$} \\
H. germanica n.a. & 12.58 & 0.371 & -13.56 & 1.091 \\
5.7 D & 45528.61 & 14.613 & 1153.00 & 2.351 \\
7.1 D & 216695.58 & 44.464 & 10361.59 & 11.271 \\
$5.5 \mathrm{P}$ & 32011.61 & 15.824 & 1627.25 & 4.389
\end{tabular}

\begin{tabular}{|c|c|c|c|c|c|c|c|c|c|c|c|}
\hline food source & $\mathrm{T}$ & treatment & $\mathrm{Nr} / \mathrm{Ind}$ & weight [mg] & & d $15 N / 14 N$ & $\mathrm{AT} \% 15 \mathrm{~N} / 14 \mathrm{~N}$ & $\mathrm{~d} 13 \mathrm{C} / 12 \mathrm{C} \mathrm{AT} \% 1$ & $13 \mathrm{C} / 12 \mathrm{C}$ & $\mu \mathrm{g} N$ & $\mu \mathrm{g} C$ \\
\hline $5.7 \mathrm{D}$ & $15^{\circ} \mathrm{C}$ & $24 \mathrm{hrs}$ fed & & 48 & 1.250 & 425.11 & 0.521 & 3.82 & 1.110 & 8.85 & 51.58 \\
\hline $5.7 \mathrm{D}$ & $15^{\circ} \mathrm{C}$ & $24 \mathrm{hrs}$ fed & & 52 & 1.277 & 416.26 & 0.518 & 3.25 & 1.109 & 8.79 & 52.80 \\
\hline $5.7 \mathrm{D}$ & $15^{\circ} \mathrm{C}$ & $24 \mathrm{hrs}$ fed & & 53 & 1.289 & - & - & - & - & - & - \\
\hline $5.7 \mathrm{D}$ & $20^{\circ} \mathrm{C}$ & $24 \mathrm{hrs}$ fed & & 50 & 0.963 & 328.42 & 0.486 & -0.42 & 1.105 & 7.75 & 43.83 \\
\hline $5.7 \mathrm{D}$ & $20^{\circ} \mathrm{C}$ & $24 \mathrm{hrs}$ fed & & 53 & 1.284 & 323.13 & 0.484 & -0.54 & 1.105 & 8.61 & 51.50 \\
\hline $5.7 \mathrm{D}$ & $20^{\circ} \mathrm{C}$ & $24 \mathrm{hrs} \mathrm{fed}$ & & 56 & 1.353 & 335.27 & 0.489 & 0.39 & 1.106 & 8.74 & 52.34 \\
\hline $5.7 \mathrm{D}$ & $25^{\circ} \mathrm{C}$ & $24 \mathrm{hrs}$ fed & & 48 & 0.972 & 257.92 & 0.461 & -2.90 & 1.102 & 8.85 & 49.25 \\
\hline $5.7 \mathrm{D}$ & $25^{\circ} \mathrm{C}$ & $24 \mathrm{hrs}$ fed & & 54 & 1.301 & 291.73 & 0.473 & -3.30 & 1.102 & 9.19 & 53.82 \\
\hline $5.7 \mathrm{D}$ & $25^{\circ} \mathrm{C}$ & $24 \mathrm{hrs}$ fed & & 50 & 1.176 & 385.61 & 0.507 & 2.53 & 1.108 & 7.89 & 45.38 \\
\hline $5.7 \mathrm{D}$ & $15^{\circ} \mathrm{C}$ & 24 hrs starved & & 49 & 1.052 & 230.04 & 0.450 & -4.70 & 1.101 & 7.58 & 42.71 \\
\hline $5.7 \mathrm{D}$ & $15^{\circ} \mathrm{C}$ & 24 hrs starved & & 61 & - & - & - & - & - & - & - \\
\hline $5.7 \mathrm{D}$ & $15^{\circ} \mathrm{C}$ & 24 hrs starved & & 53 & 1.192 & 211.71 & 0.444 & -5.63 & 1.099 & 8.70 & 51.81 \\
\hline $5.7 \mathrm{D}$ & $20^{\circ} \mathrm{C}$ & 24 hrs starved & & 48 & 1.108 & 241.56 & 0.455 & -5.04 & 1.100 & 7.44 & 41.11 \\
\hline $5.7 \mathrm{D}$ & $20^{\circ} \mathrm{C}$ & 24 hrs starved & & 45 & 1.099 & 254.99 & 0.459 & -5.81 & 1.099 & 7.96 & 43.16 \\
\hline $5.7 \mathrm{D}$ & $20^{\circ} \mathrm{C}$ & $24 \mathrm{hrs}$ starved & & 44 & 1.202 & 234.45 & 0.452 & -6.98 & 1.098 & 7.39 & 42.01 \\
\hline $5.7 \mathrm{D}$ & $25^{\circ} \mathrm{C}$ & 24 hrs starved & & 49 & 1.217 & 337.63 & 0.490 & -3.98 & 1.101 & 7.70 & 39.16 \\
\hline $5.7 \mathrm{D}$ & $25^{\circ} \mathrm{C}$ & 24 hrs starved & & 39 & 1.057 & 321.86 & 0.484 & -4.15 & 1.101 & 7.18 & 40.77 \\
\hline $5.7 \mathrm{D}$ & $25^{\circ} \mathrm{C}$ & 24 hrs starved & & 43 & 1.235 & 374.66 & 0.503 & -2.93 & 1.102 & 6.66 & 36.67 \\
\hline $7.1 \mathrm{D}$ & $15^{\circ} \mathrm{C}$ & $24 \mathrm{hrs} \mathrm{fed}$ & & 55 & 1.411 & 496.43 & 0.547 & 35.23 & 1.144 & 9.48 & 55.00 \\
\hline $7.1 \mathrm{D}$ & $15^{\circ} \mathrm{C}$ & $24 \mathrm{hrs}$ fed & & 52 & 1.158 & 619.09 & 0.592 & 50.35 & 1.161 & 9.60 & 57.15 \\
\hline $7.1 \mathrm{D}$ & $15^{\circ} \mathrm{C}$ & $24 \mathrm{hrs}$ fed & & 53 & 1.417 & 481.34 & 0.542 & 41.18 & 1.151 & 8.80 & 51.30 \\
\hline $7.1 \mathrm{D}$ & $20^{\circ} \mathrm{C}$ & $24 \mathrm{hrs}$ fed & & 49 & 1.373 & 1004.73 & 0.732 & 94.37 & 1.209 & 9.01 & 50.38 \\
\hline $7.1 \mathrm{D}$ & $20^{\circ} \mathrm{C}$ & $24 \mathrm{hrs} \mathrm{fed}$ & & 50 & 0.907 & 1148.04 & 0.784 & 113.35 & 1.229 & 7.48 & 42.49 \\
\hline $7.1 \mathrm{D}$ & $20^{\circ} \mathrm{C}$ & $24 \mathrm{hrs}$ fed & & 49 & 1.413 & 1017.81 & 0.737 & 108.25 & 1.224 & 9.49 & 54.69 \\
\hline $7.1 \mathrm{D}$ & $25^{\circ} \mathrm{C}$ & $24 \mathrm{hrs}$ fed & & 51 & 1.243 & 853.93 & 0.677 & 80.93 & 1.194 & 8.90 & 48.30 \\
\hline $7.1 \mathrm{D}$ & $25^{\circ} \mathrm{C}$ & $24 \mathrm{hrs}$ fed & & 48 & 1.195 & 934.13 & 0.706 & 83.27 & 1.197 & 7.60 & 43.78 \\
\hline $7.1 \mathrm{D}$ & $25^{\circ} \mathrm{C}$ & $24 \mathrm{hrs}$ fed & & 53 & 1.243 & 764.11 & 0.645 & 65.33 & 1.177 & 8.23 & 46.30 \\
\hline $7.1 \mathrm{D}$ & $15^{\circ} \mathrm{C}$ & 24 hrs starved & & 50 & 1.128 & 432.69 & 0.524 & 12.73 & 1.120 & 7.28 & 40.52 \\
\hline $7.1 \mathrm{D}$ & $15^{\circ} \mathrm{C}$ & 24 hrs starved & & 46 & 1.166 & 465.91 & 0.536 & 16.65 & 1.124 & 7.27 & 43.98 \\
\hline $7.1 \mathrm{D}$ & $15^{\circ} \mathrm{C}$ & 24 hrs starved & & 59 & 1.251 & 416.39 & 0.518 & 15.44 & 1.123 & 8.80 & 51.35 \\
\hline $7.1 \mathrm{D}$ & $20^{\circ} \mathrm{C}$ & 24 hrs starved & & 49 & 1.160 & 555.79 & 0.569 & 23.69 & 1.132 & 7.88 & 44.51 \\
\hline $7.1 \mathrm{D}$ & $20^{\circ} \mathrm{C}$ & 24 hrs starved & & 48 & 1.209 & 715.38 & 0.627 & 35.48 & 1.144 & 7.74 & 42.10 \\
\hline $7.1 \mathrm{D}$ & $20^{\circ} \mathrm{C}$ & $24 \mathrm{hrs}$ starved & & 50 & 1.280 & 771.33 & 0.647 & 34.82 & 1.144 & 8.32 & 45.05 \\
\hline $7.1 \mathrm{D}$ & $25^{\circ} \mathrm{C}$ & 24 hrs starved & & 53 & 1.335 & 797.43 & 0.657 & 33.54 & 1.142 & 8.08 & 45.90 \\
\hline $7.1 \mathrm{D}$ & $25^{\circ} \mathrm{C}$ & $24 \mathrm{hrs}$ starved & & 48 & 1.144 & 855.89 & 0.678 & 51.27 & 1.162 & 7.87 & 41.64 \\
\hline $7.1 \mathrm{D}$ & $25^{\circ} \mathrm{C}$ & $24 \mathrm{hrs}$ starved & & 54 & 1.230 & 845.19 & 0.674 & 43.79 & 1.154 & 8.00 & 45.05 \\
\hline $5.5 \mathrm{P}$ & $15^{\circ} \mathrm{C}$ & $24 \mathrm{hrs}$ fed & & 50 & 1.469 & 541.16 & 0.564 & 64.97 & 1.177 & 8.27 & 49.43 \\
\hline $5.5 \mathrm{P}$ & $15^{\circ} \mathrm{C}$ & $24 \mathrm{hrs}$ fed & & 51 & 1.359 & 618.81 & 0.592 & 68.13 & 1.180 & 9.73 & 56.12 \\
\hline $5.5 \mathrm{P}$ & $15^{\circ} \mathrm{C}$ & $24 \mathrm{hrs} \mathrm{fed}$ & & 51 & 1.398 & 559.13 & 0.570 & 60.14 & 1.171 & 9.53 & 54.99 \\
\hline $5.5 \mathrm{P}$ & $20^{\circ} \mathrm{C}$ & $24 \mathrm{hrs}$ fed & & 52 & 1.172 & 598.98 & 0.585 & 58.26 & 1.169 & 10.87 & 60.65 \\
\hline $5.5 \mathrm{P}$ & $20^{\circ} \mathrm{C}$ & $24 \mathrm{hrs}$ fed & & 52 & 1.363 & 576.87 & 0.577 & 62.15 & 1.174 & 10.05 & 52.00 \\
\hline $5.5 \mathrm{P}$ & $20^{\circ} \mathrm{C}$ & $24 \mathrm{hrs}$ fed & & 51 & 1.117 & 484.73 & 0.543 & 57.47 & 1.168 & 10.69 & 53.97 \\
\hline $5.5 \mathrm{P}$ & $25^{\circ} \mathrm{C}$ & $24 \mathrm{hrs}$ fed & & 47 & 1.012 & 439.52 & 0.527 & 32.98 & 1.142 & 9.38 & 46.52 \\
\hline $5.5 \mathrm{P}$ & $25^{\circ} \mathrm{C}$ & $24 \mathrm{hrs}$ fed & & 49 & 1.114 & 461.60 & 0.535 & 33.99 & 1.143 & 9.49 & 46.77 \\
\hline $5.5 \mathrm{P}$ & $25^{\circ} \mathrm{C}$ & $24 \mathrm{hrs}$ fed & & 49 & 1.127 & 403.72 & 0.514 & 34.21 & 1.143 & 10.93 & 54.56 \\
\hline $5.5 \mathrm{P}$ & $15^{\circ} \mathrm{C}$ & 24 hrs starved & & 48 & 1.246 & 364.87 & 0.500 & 44.50 & 1.154 & 7.43 & 40.48 \\
\hline $5.5 \mathrm{P}$ & $15^{\circ} \mathrm{C}$ & 24 hrs starved & & 52 & 1.531 & 397.28 & 0.511 & 47.21 & 1.157 & 9.18 & 52.64 \\
\hline $5.5 \mathrm{P}$ & $15^{\circ} \mathrm{C}$ & 24 hrs starved & & 53 & 1.508 & 351.37 & 0.495 & 48.17 & 1.158 & 9.13 & 50.88 \\
\hline $5.5 \mathrm{P}$ & $20^{\circ} \mathrm{C}$ & 24 hrs starved & & 51 & 1.098 & 395.17 & 0.511 & 43.51 & 1.153 & 10.00 & 49.00 \\
\hline $5.5 \mathrm{P}$ & $20^{\circ} \mathrm{C}$ & 24 hrs starved & & 54 & 1.019 & 365.61 & 0.500 & 40.76 & 1.150 & 9.27 & 45.91 \\
\hline $5.5 \mathrm{P}$ & $20^{\circ} \mathrm{C}$ & $24 \mathrm{hrs}$ starved & & 55 & 1.322 & 362.58 & 0.499 & 31.43 & 1.140 & 9.90 & 48.50 \\
\hline $5.5 \mathrm{P}$ & $25^{\circ} \mathrm{C}$ & 24 hrs starved & & 54 & 1.046 & 289.12 & 0.472 & 18.97 & 1.126 & 9.62 & 50.96 \\
\hline $5.5 \mathrm{P}$ & $25^{\circ} \mathrm{C}$ & $24 \mathrm{hrs}$ starved & & 39 & 0.952 & 333.26 & 0.488 & 22.21 & 1.130 & 7.14 & 36.69 \\
\hline $5.5 \mathrm{P}$ & $25^{\circ} \mathrm{C}$ & 24 hrs starved & & 57 & 1.327 & 313.36 & 0.481 & 19.12 & 1.127 & 10.97 & 52.50 \\
\hline
\end{tabular}


Table S2. Raw data of GC-IRMS of water samples.

\begin{tabular}{|c|c|c|c|c|c|}
\hline & food source & $\mathrm{T}$ & avg $\mathrm{d} 13 \mathrm{C} / 12 \mathrm{C}$ & avg AT\% $13 \mathrm{C} / 12 \mathrm{C}$ & ppm CO2 \\
\hline $\mathrm{H}_{3} \mathrm{PO}_{4}$ & & - & -18.734 & 1.0852 & 83.85 \\
\hline \multirow[t]{25}{*}{$\mathrm{H}_{3} \mathrm{PO}_{4}+\mathrm{SW}$} & & - & -1.537 & 1.1040 & 9162.91 \\
\hline & $5.7 \mathrm{D}$ & $15^{\circ} \mathrm{C}$ & -1.135 & 1.1044 & 9398.27 \\
\hline & $5.7 \mathrm{D}$ & $15^{\circ} \mathrm{C}$ & -1.250 & 1.1043 & 8406.95 \\
\hline & $5.7 \mathrm{D}$ & $15^{\circ} \mathrm{C}$ & -1.156 & 1.1044 & 9883.24 \\
\hline & $5.7 \mathrm{D}$ & $20^{\circ} \mathrm{C}$ & -0.870 & 1.1047 & 9184.63 \\
\hline & $5.7 \mathrm{D}$ & $20^{\circ} \mathrm{C}$ & -1.162 & 1.1044 & 7778.55 \\
\hline & $5.7 \mathrm{D}$ & $20^{\circ} \mathrm{C}$ & -0.994 & 1.1046 & 8489.17 \\
\hline & $5.7 \mathrm{D}$ & $25^{\circ} \mathrm{C}$ & -0.963 & 1.1046 & 8829.57 \\
\hline & $5.7 \mathrm{D}$ & $25^{\circ} \mathrm{C}$ & -1.047 & 1.1045 & 9614.38 \\
\hline & $7.1 \mathrm{D}$ & $15^{\circ} \mathrm{C}$ & -0.887 & 1.1047 & 9472.19 \\
\hline & $7.1 \mathrm{D}$ & $15^{\circ} \mathrm{C}$ & -0.756 & 1.1048 & 9247.92 \\
\hline & $7.1 \mathrm{D}$ & $15^{\circ} \mathrm{C}$ & -1.060 & 1.1045 & 9794.93 \\
\hline & $7.1 \mathrm{D}$ & $20^{\circ} \mathrm{C}$ & -0.941 & 1.1046 & 6902.25 \\
\hline & $7.1 \mathrm{D}$ & $20^{\circ} \mathrm{C}$ & -0.762 & 1.1048 & 7843.66 \\
\hline & $7.1 \mathrm{D}$ & $20^{\circ} \mathrm{C}$ & -0.910 & 1.1047 & 7959.04 \\
\hline & $7.1 \mathrm{D}$ & $25^{\circ} \mathrm{C}$ & -0.306 & 1.1053 & 7818.50 \\
\hline & $7.1 \mathrm{D}$ & $25^{\circ} \mathrm{C}$ & -0.465 & 1.1052 & 9206.42 \\
\hline & $5.7 \mathrm{P}$ & $15^{\circ} \mathrm{C}$ & -0.861 & 1.1047 & 7659.74 \\
\hline & $5.7 \mathrm{P}$ & $15^{\circ} \mathrm{C}$ & -0.971 & 1.1046 & 9208.57 \\
\hline & $5.7 \mathrm{P}$ & $15^{\circ} \mathrm{C}$ & -0.655 & 1.1049 & 9055.14 \\
\hline & $5.7 \mathrm{P}$ & $20^{\circ} \mathrm{C}$ & -0.536 & 1.1051 & 9910.84 \\
\hline & $5.7 \mathrm{P}$ & $20^{\circ} \mathrm{C}$ & -0.538 & 1.1051 & 8473.16 \\
\hline & $5.7 \mathrm{P}$ & $20^{\circ} \mathrm{C}$ & -0.691 & 1.1049 & 9219.05 \\
\hline & $5.7 \mathrm{P}$ & $25^{\circ} \mathrm{C}$ & -0.351 & 1.1053 & 7268.07 \\
\hline & $5.7 \mathrm{P}$ & $25^{\circ} \mathrm{C}$ & -0.664 & 1.1049 & 7246.05 \\
\hline $\mathrm{He}$ & & & & & 0 \\
\hline
\end{tabular}




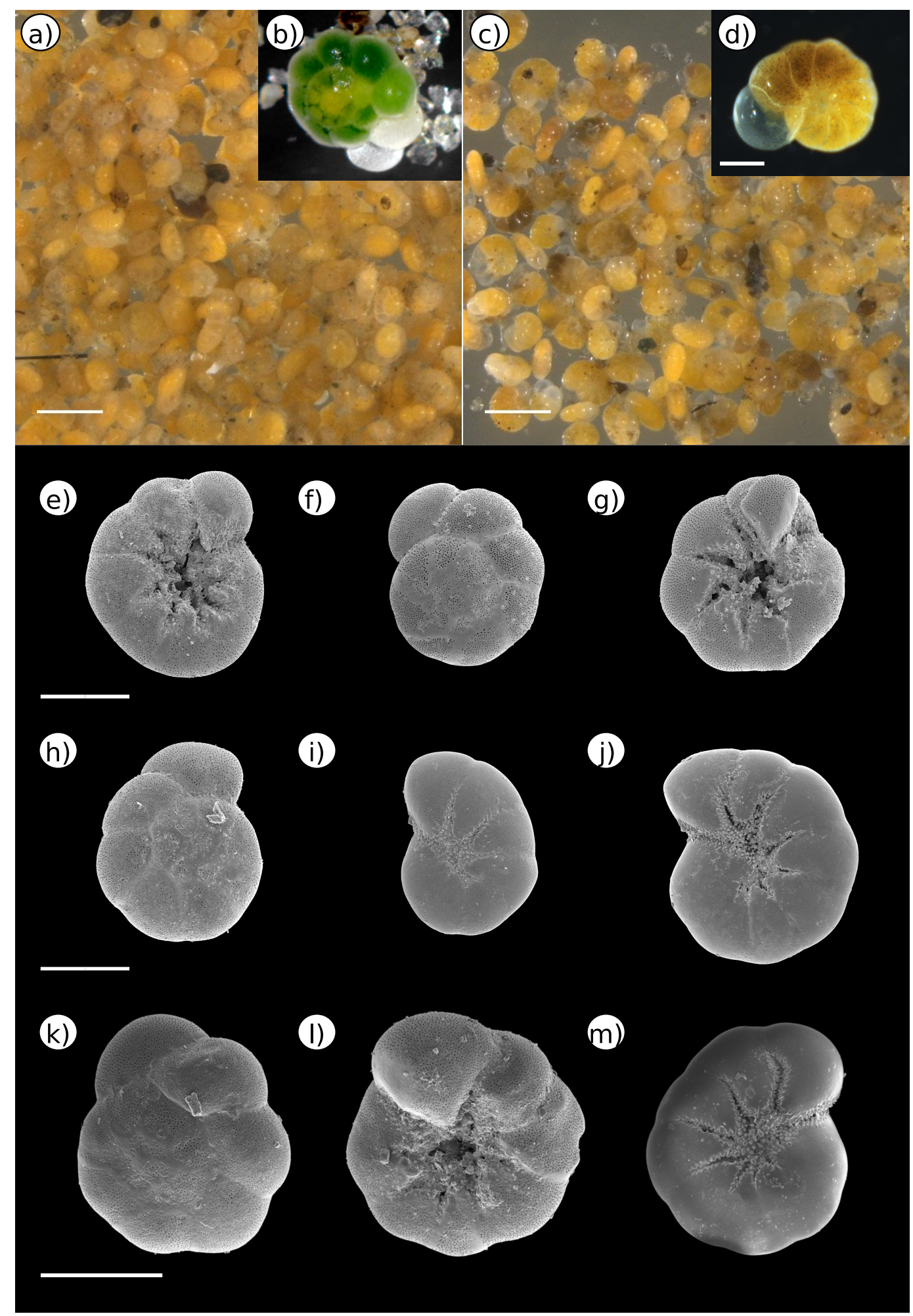

Supplementary Figure 1. a) Light microscope image of fresh picked $A$. tepida specimens (scale bar = $500 \mu \mathrm{m}$ ). b) A. tepida after feeding on fresh microalgae. c) Fresh picked $H$. germanica specimens (scale bar $=500 \mu \mathrm{m})$. d) $H$. germanica individual (scale bar $=200 \mu \mathrm{m})$. e)-h) SEM images of $A$. tepida collected in 2014 at the sampling location of this study (scale bar $=200 \mu \mathrm{m}$ ). i)-j) H. germanica collected in 2014 at the sampling location of this study (scale bar $=200 \mu \mathrm{m})$. k)-1) A. tepida collected in 2016 at the sampling location of this study (scale bar $=200 \mu \mathrm{m}$ ). m) H. germanica collected in 2016 at the sampling location of this study (scale bar $=200 \mu \mathrm{m}$ ). 